\title{
Role of magnetic resonance imaging in characterization of central nervous system lesions in pediatric patients with leukemia and post-treatment complications
}

\author{
Eman M. Amer ${ }^{1 *}$ (D) Ahmed F. Youssef ${ }^{2}$, Marwa A. Romeih ${ }^{3}$, Ayda A. Youssef ${ }^{4}$ and Hamada M. Khater ${ }^{2}$
}

\begin{abstract}
Background: Leukemia is one of the most common fatal diseases in pediatric oncology. Recently, advances in drug therapy have improved the prognosis of acute leukemia with event-free survival of up to 60\%; however, complications and adverse effects of the disease and anti-leukemic treatment have also increased. The CNS complications of leukemia can be classified into those that developed directly or indirectly from the underlying leukemic process and those that can be related to antileukemic therapy. MRI had improved early detection of CNS complications and proper management. The study aims to characterize the MRI findings caused by the leukemic involvement of CNS structures and treatment-associated CNS complications and assess its value in early management and avoidance of long-term side effects.

Results: The patient's age ranged from 2 to 18 years with different types of leukemia classified regarding the time of presentation as pretreatment, during treatment phases, and post-treatment. Different MRI abnormalities were recorded and clinically correlated.

Conclusion: The neurological complications of leukemia have common presenting symptoms but varying imaging abnormalities. To reach the correct diagnosis, the presenting signs, symptoms, and laboratory data must be considered along with the radiologic findings. A diagnostic algorithm using conventional, post-contrast MRI, MR venography, along with diffusion-weighted MRI was of great value in early detection and differentiation of different CNS lesions detected in pediatric patients with leukemia and post-treatment CNS complications.
\end{abstract}

Keywords: Magnetic resonance imaging, Leukemia, CNS

\section{Background}

Leukemia is a heterogeneous group of disorders, caused by a neoplastic transformation in blood cell precursors during their differentiation in bone marrow [1]. It is the most prevalent malignancy in children and represents nearly $30 \%$ of all malignancies, with $75 \%$ of cases corresponding to acute lymphoblastic leukemia (ALL). The prevalence of all forms of leukemia is more in children under 5 years of age and decreases significantly with

\footnotetext{
* Correspondence: eman.amer83@gmail.com

${ }^{1}$ Tanta Cancer Center, El Nadi Street, Tanta, Egypt

Full list of author information is available at the end of the article
}

advancing age. Diagnosis is made through a wide range of clinical and laboratory findings [2]. The central nervous system (CNS) complications of leukemia were rarely seen due to the rapid course of the disease. But now, therapeutic advances have improved the prognosis of acute leukemia with a survival rate up to $60 \%$ [3]. The prophylactic therapy of the CNS includes intrathecal methotrexate, high-dose chemotherapy, radiotherapy, or a combination of any of these. However, patients with ALL can present with neurological complications as a result of this therapy and the underlying systemic effects of leukemia [4]. The neurological complications of 


\section{Type of Leukemia}

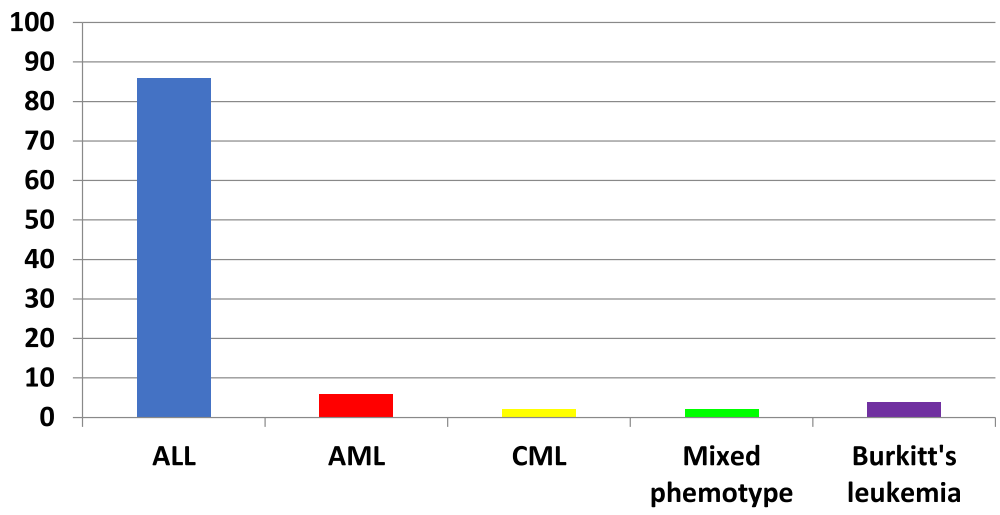

Fig. 1 Distribution of leukemia types among the studied group

leukemia can be divided into (a) infiltration of the CNS by leukemic cells or (b) secondary complications due to the disease, and/or its therapy. The disease itself may involve the leptomeninges, brain parenchyma, or cerebral vasculature. CNS complications related to treatment include white matter lesions, small-vessel calcifications, cerebrovascular disorders, secondary tumors and infections, atrophic brain changes, endocrinopathies, and/or neurocognitive deficits caused by the "late effects" of their treatment [5].

Diagnosing CNS lesions in leukemia is often burdensome due to a variable set of causes; there are no pathognomonic imaging features for CNS lesions in leukemia, and the histological verification of a brain biopsy remains the gold standard for diagnosis. As many CNS lesions in leukemia are curable, early diagnosis is important for their proper management. MRI is known for its superior soft-tissue imaging; it was observed that MRI aided the characterization of CNS lesions caused by the leukemic involvement of CNS structures and treatment-associated CNS complications and thus will lead to early management and prevention or minimizing the side effects of the disease [6]. The purpose of this study is to analyze the MRI imaging features of these complications and interpreting these scans in view of clinical data to assess its value in early management.

\section{Methods}

\section{Study patients}

This prospective study was performed during the period from April 2018 to June 2020 which included 50 child patients with leukemia (30 males and 20 females) presented with neurological manifestations. The inclusion criteria were pediatric age group (up to 18 years) and confirmed cases of leukemia presented with neurological manifestations, while the exclusion criteria were patient over 18 years old and pediatric patient with leukemia but with no neurological manifestations. Patients included in the study were subjected to history taking, including age, sex, type of leukemia, clinical picture, and stage of treatment.

Time of development of CNS manifestations

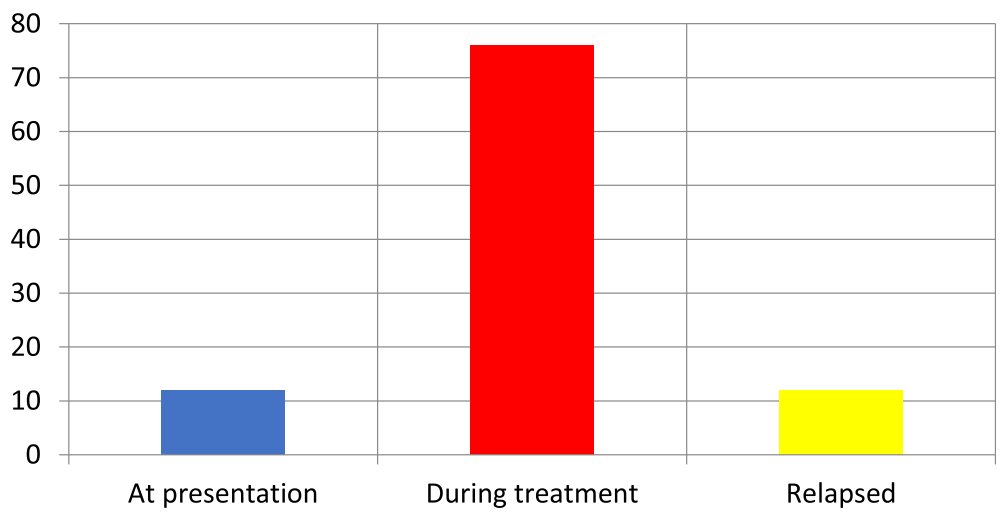

Fig. 2 Time of development of CNS manifestations among the studied group 
Table 1 Phase of treatment at study time

\begin{tabular}{lll}
\hline Phase of treatment & No. & Percentage \\
\hline Induction & $\mathbf{1 8}$ & $48.6 \%$ \\
Consolidation & $\mathbf{5}$ & $13.5 \%$ \\
Maintenance & 14 & $38 \%$ \\
\hline
\end{tabular}

\section{Image acquisition}

All patients included in this study underwent:

(1) Conventional MRI 1.5 T, superconducting MR imager (Magnotom ESPREE 1.5 T, Erlangen, Semines, Germany). All the cases were examined in the supine position with standard circularly polarized head coil using the following sequences:

Axial T1WI (450/12 ms) TR/TE (repetition time/echo time) spin echo.

Axial T2WI (4540/96 ms) TR/TE spin echo.

Axial FALIR (9000/116/2500 ms) TR/TE/TI).

Sagittal T1WI (430/10 ms) TR/TE spin echo.

Five-millimeter-section thickness and $256 \times 256$ matrix size.

(2) MR diffusion imaging: Axial diffusion-weighted imaging was performed and the parameters used were $\mathrm{TR}=3160 \mathrm{~ms}$, TE $=100 \mathrm{~ms}$, FOV $=200 \mathrm{~mm}$, acquisition matrix $=25677$ pixels, slice thickness $=4$ $\mathrm{mm}$, with $0.5 \mathrm{~mm}$ gap. The ADC value was calculated through an automatically performed ADC map.

(3) MR venography (MRV): Time-of-flight MR venography was performed in the patient with cerebrovascular disorders. The parameters used were as follows: 30/9/1; a field of view, 22-25 cm; matrix $219 \times 250$; flip angle, 50; section thickness, 3 $\mathrm{mm}$; section slap thickness, 3-7.5. Ten sections.

(4) Intravenous administration of contrast was used in 13 cases suspected with leukemic infiltration versus infection. Gadolinium-DTPA $(0.3 \mathrm{mg} / \mathrm{kg})$ and contrast-enhanced T1WI in axial, sagittal, and coronal planes were obtained.

In 20 cases of the studied patients, we needed deep sedation or general anesthesia to minimize motion artifacts during MRI examinations.

\section{Interpretation of MRI findings}

The MRI findings in all cases were interpreted by two consultants of radiology in our department; each had more than 10 years of experience, conjoint reading.

The study was done after the approval of the Research and Ethical Committee. Informed consent from each child's guardian was obtained before performing the study.

\section{Results}

The current study included 50 patients. Their ages at the time of the study ranged from 2-18 years. Males represented the $60 \%$, while females represented the $40 \%$. The patients were classified according to the type of leukemia into acute lymphocytic leukemia (ALL) (86\%), acute myeloid leukemia (AML) (6\%), mixed phenotype leukemia (2\%), Burkitt's leukemia (4\%), and chronic myeloid leukemia (CML) (2\%) (Fig. 1). The most common symptoms recorded in our patients were headache, seizure, limb weakness, diminished vision, nausea, vomiting, and fever.

Regarding the time of development of CNS manifestations, $76 \%$ of the studied patients had CNS manifestation during treatment phases, while $12 \%$ at the time of presentation before the start of treatment and $12 \%$ were relapsed disease post-end of treatment (Fig. 2).

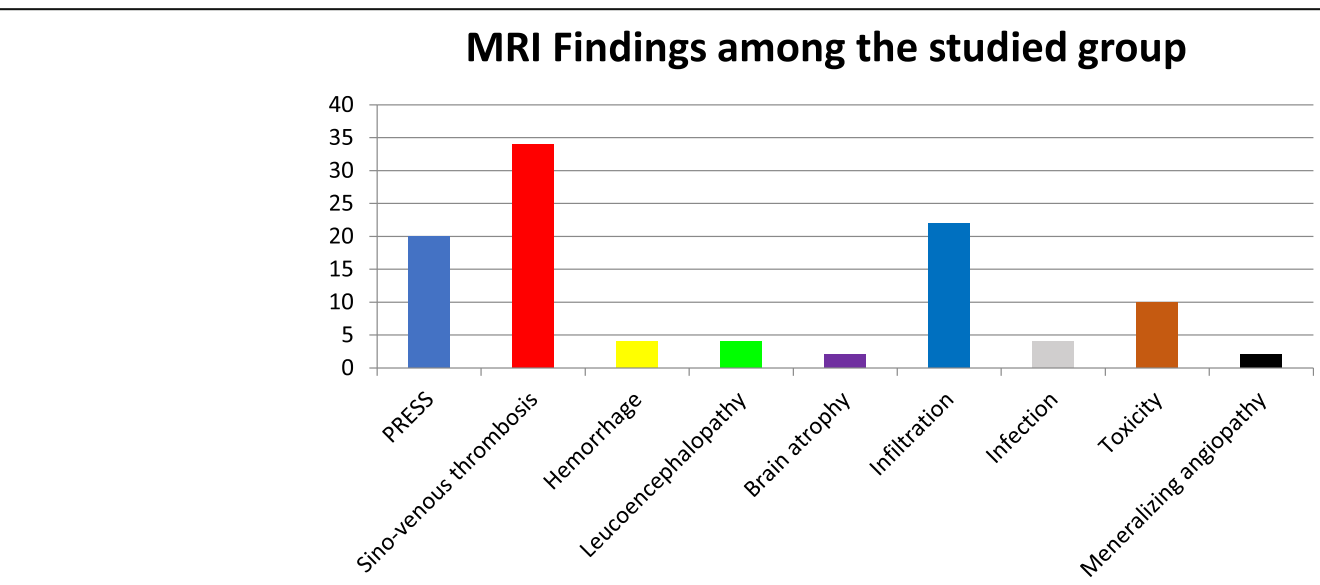

Fig. 3 MR findings among the studied group 
Table 2 Frequency of the MRI diagnoses within the phases of presentation

\begin{tabular}{llllll}
\hline $\begin{array}{l}\text { Phase of treatment } \\
\text { MRI abnormality }\end{array}$ & Induction & Consolidation & Maintenance & $\begin{array}{l}\text { At } \\
\text { presentation }\end{array}$ \\
\hline PRES & 7 & 1 & 1 & - & Relapse \\
Hemorrhage & - & - & 1 & 1 & - \\
Sinovenous thrombosis & 7 & 1 & 1 & - & - \\
Leucoencephalopathy & - & - & - & - & - \\
Brain atrophy & - & - & - & 5 & - \\
Infection & 2 & - & - & 3 \\
Infiltration & 3 & - & 1 & - \\
Toxicity & & 3 & 1 & - \\
Mineralizing angiopathy & - & - & &
\end{tabular}

Regarding the phase of treatment at study time, $48.6 \%$ were at the induction phase, $38 \%$ at the maintenance phase, while $13.5 \%$ at the consolidation phase (Table 1).

MR abnormalities among the studied group were collected and classified to posterior reversible encephalopathy syndrome (PRES) 20\%, sinovenous thrombosis $34 \%$, hemorrhage $4 \%$, infiltration $22 \%$, methotrexate (MTX) toxicity $10 \%$, infection $4 \%$, mineralizing angiopathy $2 \%$, leucoencephalopathy $2 \%$, and brain atrophy $2 \%$ (Fig. 3).

The frequency of different MRI abnormalities regarding to treatment phase was depicted in Table 2.

PRES lesions were common at the induction phase $(80 \%)$, while a small percentage at the consolidation and the maintenance phase in $10 \%$ per each. PRES lesions were identified in FLAIR images, and their distribution in anatomical regions was made (Fig. 4). The most commonly involved typical regions were the occipital lobes (90\%), and this was followed by the parietal lobes $(80 \%)$, frontal lobe (50\%), and cerebellum (10\%) (Fig. 5). The other atypical regions involved were the basal ganglia, thalamus, and brainstem in $10 \%$ per each (Fig. 6). All patients with cerebral lesions had bilateral distribution apart from one patient who had unilateral distribution affecting the left frontal lobe only. Follow-up study of that patient after a few days revealed new lesions appeared at both occipital lobes. All patients were normotensive apart from two patients who were hypotensive. In DWI, most patients showed facilitated diffusion $(80 \%)$ and two patients (20\%) had a restricted DWI pattern.

Hemorrhage was detected in two patients; one patient who was at the maintenance phase showed left frontal intra-axial hematoma, and the other patient who was at the time of presentation had multiple parenchymal hemorrhagic foci.

Cerebral venous sinus thrombosis was detected in 17 patients. It was seen mainly at the maintenance phase (53\%) and at the induction phase (41\%); only 5\% was seen at the consolidation phase (Table 3). The superior sagittal sinus was the commonest involved (76\%), followed by the transverse sinuses which were involved in $41 \%$. The left transverse sinus was more commonly involved in six cases, while the right transverse sinus was involved in one case (Fig. 7).

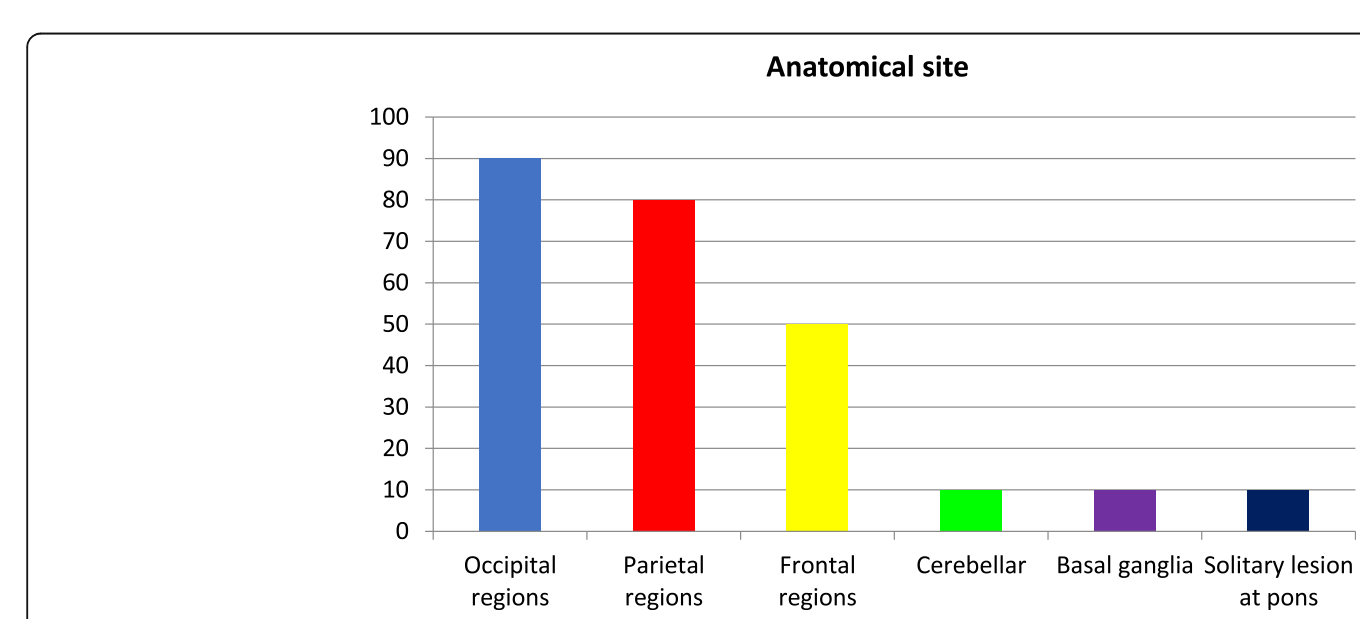

Fig. 4 Confirmed cases of PRES with involvement of the typical and atypical regions of the brain 

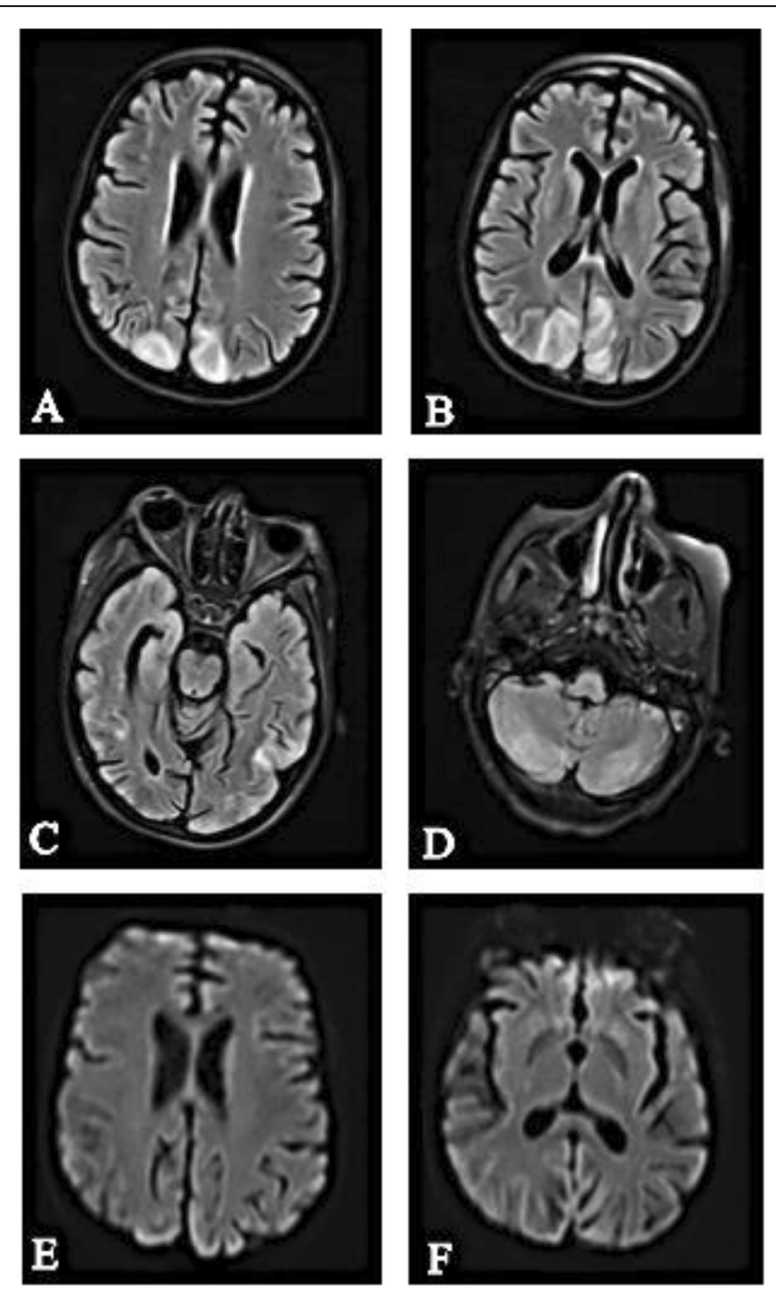

Fig. 5 Typical pattern of posterior reversible encephalopathy syndrome. Female patient, 13 years old, treated for ALL at the maintenance phase presented with headache and seizure axial MRI of the brain. a-d FLAIR images show bilateral cortical and subcortical areas of bright signal involving both the parietooccipital regions and both the cerebellar hemisphere $(\mathbf{e}, \mathbf{f})$. DWI of the patient show no evidence of diffusion restriction

Cerebral infection represented $4 \%$ of the studied cases. In the current study, two patients developed a cerebral infection with fungal aspergillosis seen at the induction phase. The first patient showed multiple cerebral masses, and the second patient had a focal lesion at the midbrain. In both cases, the lesions elicited an intermediate signal at T1WI with a marginal high signal. At T2WI, the core of the mass became low signal. At the DWI, the lesions showed marginal diffusion restriction, while the gradient images showed blooming of the center of the lesions. The first patient died within a few days, and the other improved with antifungal treatment. Both cases had concomitant chest fungal infection with a positive galactomannan test at the serum (Fig. 8).
Leukemic infiltration was seen at 11 cases, classified regarding the type of leukemia to seven cases with ALL (63\%), two cases with Burkitt's leukemia (18\%), one case with AML (9\%), and one case with CML (9\%) (Table 4).

As regards to the site of infiltration, the MRI findings were classified as retinal, cerebral, meningeal, orbital, skull, and maxillofacial bone infiltration (Table 5).

Three cases $(27 \%)$ had retinal infiltration presented with enhancing retinal nodules, elicited intermediate signal in T1WI, and slightly low signal in T2WI with postcontrast enhancement and diffusion restriction in DWI. Two cases (18\%) had cerebral infiltration: the first case presented with multiple foci of abnormal parenchymal signal and enhancement seen at the thalami, brain stem, and cerebellum with no diffusion restriction, and the second case showed the right parieto-occipital and the right basal ganglia area of high FLAIR signal with postcontrast enhancement. Three cases (27\%) had meningeal infiltration: one at presentation had dural thickening and enhancement seen at the frontal, temporal, and parasellar regions, the other presented with late CNS relapse had a subdural collection with cranial and spinal meningeal irregular thickening with enhancement, while the last one had intraventricular enhancing masses (Fig. 9). All cases had CSF analysis revealed blast cells in CSF. One case $(9 \%)$ had an enhancing left orbital mass at presentation proved by biopsy as leukemic infiltration (Fig. 10). Two cases (18\%) with skull bones and maxillofacial bone infiltration showed diffuse infiltrative marrow signal with periosteal reaction and periosteal soft tissue tumefaction (Fig. 11).

MTX toxicity was detected in five cases (10\%); $60 \%$ at the consolidation phase, one $20 \%$ at the maintenance phase, and $20 \%$ at the re-induction phase post-relapse. Most cases showed a rather symmetrical periventricular, centrum semi-oval foci of restricted diffusion with no abnormality at T1-T2 or FLAIR (Fig. 12).

Leuko-encephalopathy was detected in $2 \%$ at the maintenance phase presented with a periventricular sheet of a high signal at FLAIR images, classified as grade I regarding the US National Cancer Institute grading of leukoencephalopathy. Isolated atrophic brain changes were detected in $2 \%$, presented with late hematological relapse and had prominent cerebral ventricle, extra axial CSF spaces, and mild volume loss. Mineralizing angiopathy (2\%) was seen at the maintenance phase. This patient was treated for ALL. CT showed bilateral subcortical calcific foci seen at the graywhite matter interface. In MRI, the calcifications were not clearly visible. In the follow-up studies, the patient developed cortical laminar necrosis appeared as a cortical bright signal at T1WI. Those patients were treated with intrathecal chemotherapy with no history of cranial radiation. 

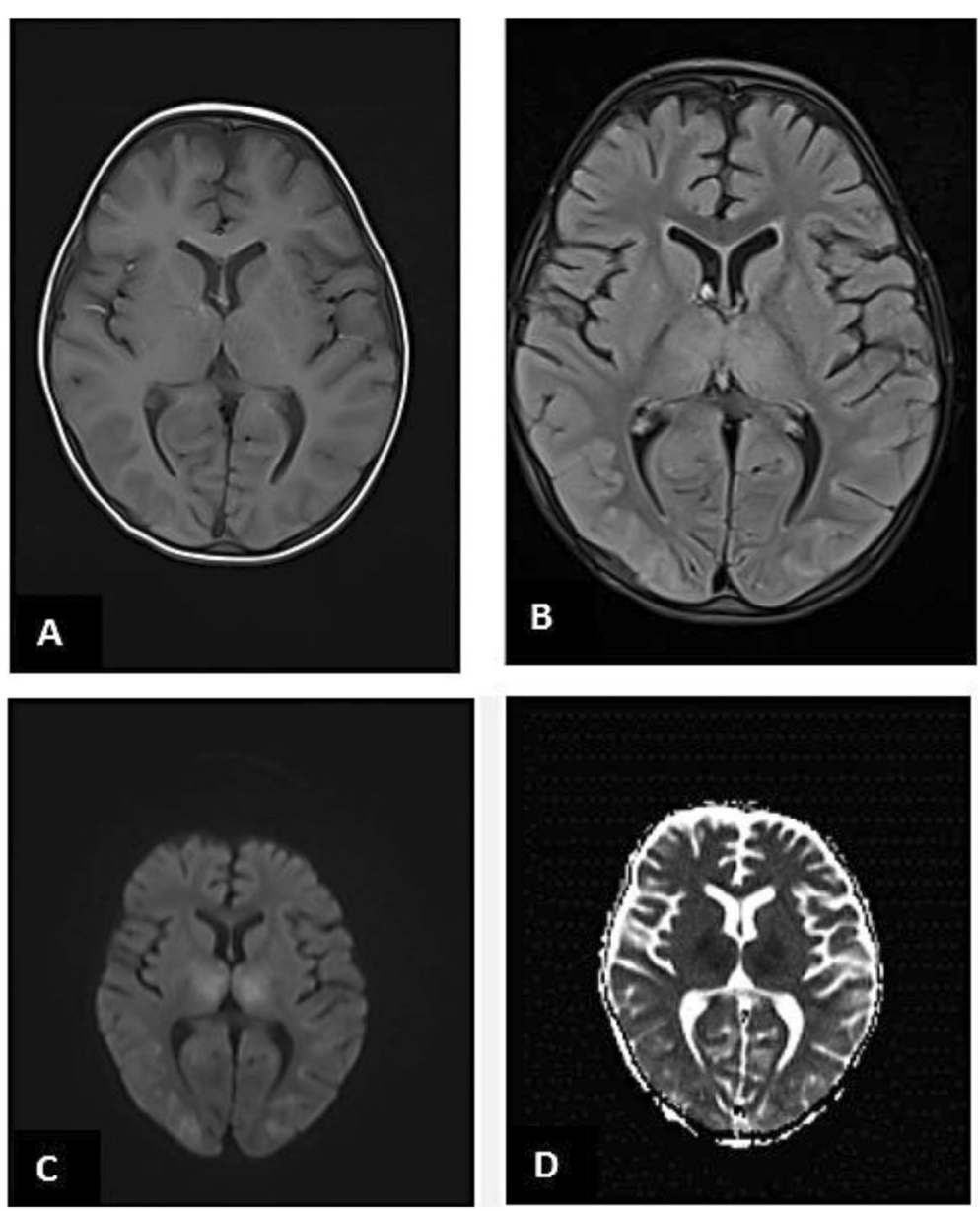

Fig. 6 Atypical pattern of posterior reversible encephalopathy syndrome. Female patient, 2 years old, diagnosed with ALL at the induction phase Axial MRI images of the brain at the level of the thalamus. a T1WI, $\mathbf{b}$ FLAIR, $\mathbf{c}$ DWI, and $\mathbf{d}$ ADC show bilateral thalamic and occipital cortical and subcortical areas of faint low signal in T1WI and faint bright signal in FLAIR images with diffusion restriction in DWI and ADC map

\section{Discussion}

Leukemia is considered one of the most common childhood cancers. Long ago, the central nervous system complications of leukemia were rarely seen because the disease was rapid and fatal. Latterly, advances in imaging techniques and treatment methods have prolonged survival, but unfortunately, the frequency of CNS complications has increased. We divide the CNS complications of leukemia into those that result directly or indirectly from the underlying leukemic process and those related to treatment side effects $[5,7]$.

Table 3 Distribution of cases with cerebral venous thrombosis regarding phase of treatment

\begin{tabular}{lll}
\hline Phase of treatment & Number of cases & Percentage \\
\hline Induction & 7 & $41 \%$ \\
Consolidation & 1 & $5 \%$ \\
Maintenance & 9 & $53 \%$ \\
\hline
\end{tabular}

We prospectively evaluated the cranial MRI of $50 \mathrm{pa}$ tients proved with leukemia ranging in age from 2 to 18 years and having CNS manifestations. Male represented $60 \%$ in our study, while females represented $40 \%$.

ALL represented $86 \%$ of our cases, while AML represented 6\%. Mixed phenotype leukemia and CML each represented 2\%, while Burkitt's leukemia represented $4 \%$. This coordinated with previous studies of Verma et al. [2] and Terwilliger et al. [7] which stated that ALL is the most common type of leukemia.

In the current study, vascular disorders were the most common complication (58\%), of which (34\%) had sinovenous thrombosis, $20 \%$ had PRES, and $4 \%$ had a parenchymal hemorrhage.

Cerebral venous sinus thrombosis was considered the most common complication seen commonly in the maintenance phase $53 \%$ followed by the induction phase $41 \%$ and a few percentages $5 \%$ in the consolidation phase. The study performed by Malhotra et al. [8] and Porto et al. [9] said that cerebro-venous thrombosis was 

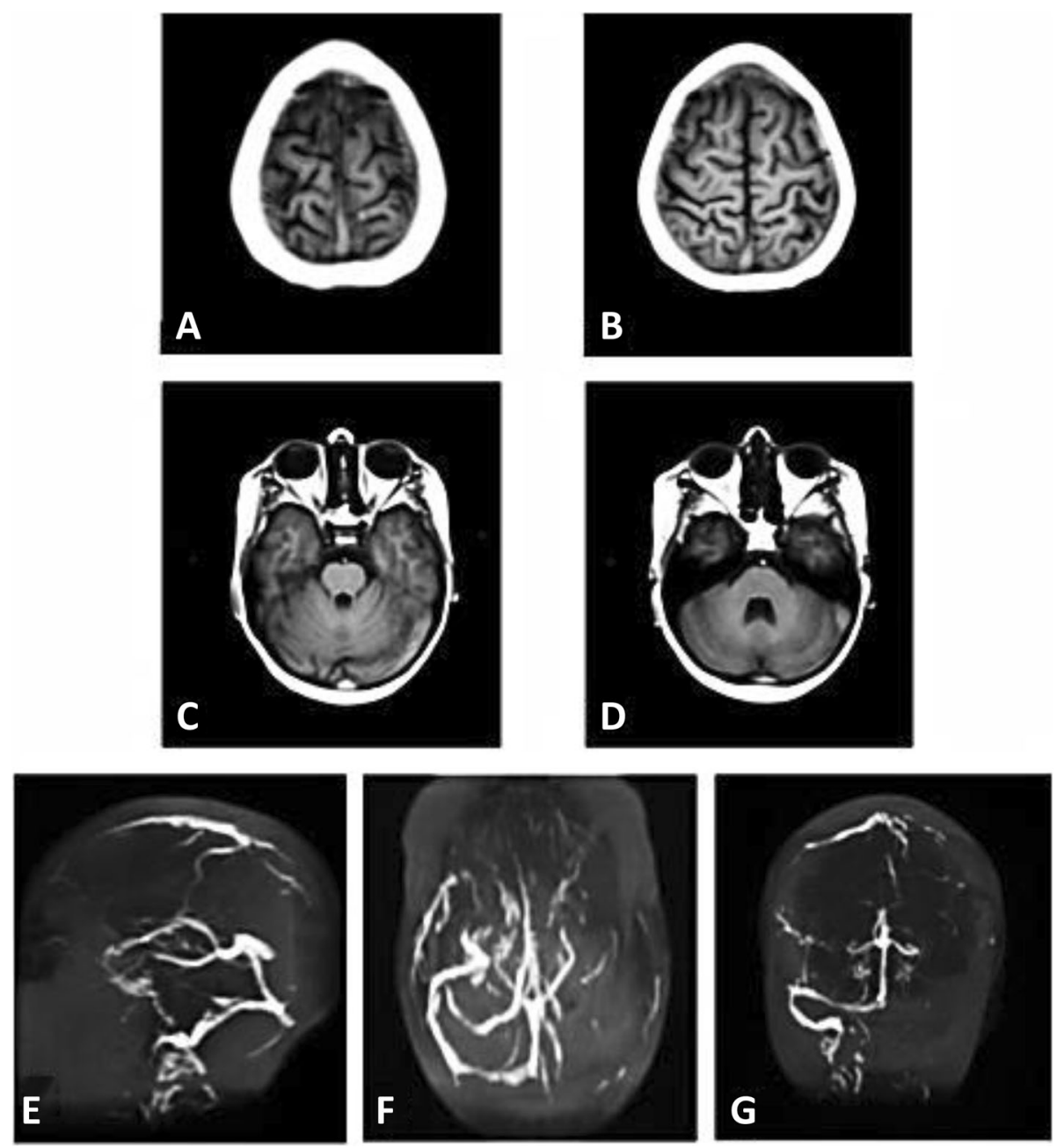

Fig. 7 Superior sagittal sinus and left transverse sinus thrombosis. Female patient, 7 years old, treated for ALL at the maintenance phase, on $L$ asparaginase treatment presented with severe headache. a-d Axial MRI of the brain. $\mathbf{a}, \mathbf{b}$ Axial T1WI at the level of frontal lobes revealed acute thrombus filling the superior sagittal sinus. $\mathbf{c}$, $\mathbf{d}$ Images axial T1WI of the same patient at the level of posterior fossa shows acute thrombus filling left transverse and left sigmoid sinuses eliciting bright signal in T1WI. e-g MIP images of MRV, sagittal (e), axial (f), and coronal (g) of the same patient show thrombosis of the posterior aspect of superior sagittal sinus, left transverse and left sigmoid sinuses

most common in the induction phase. Both studies have reported that venous sinuous thrombosis commonly happened post-exposure to L-asparaginase therapy in induction and maintenance phases. Treatment with Lasparaginase leads to the depletion of plasma proteins involved in both coagulation and fibrinolysis and has been linked to cerebrovascular complications including cortical infarcts, dural sinus thrombosis, intracerebral hemorrhage, and hemorrhagic infarcts. Cerebrovascular thrombosis or hemorrhage can also occur during antileukemic treatment as a result of leukocytosis, thrombocytopenia, sepsis, and coagulopathy [9]. This explains the sinovenous thrombosis in one of our patients in the consolidation phase apparently not related to asparaginase therapy.
For the diagnosis of venous thrombosis, the combination of conventional MR imaging with MRV could detect venous sinus thrombosis in all cases in our study. This agrees with multiple studies $[10,11]$ which stated that the combination of MR and MRV is now the method of choice because of its capability to reveal the absence of flow in the cerebral veins even with the absence of typical findings of brain infarcts. MRV can demonstrate the thrombus in the acute phase by the absence of flow which can be mistaken in conventional images for flowing blood.

PRES is a clinico-neuro-radiologic disease with distinct MR imaging findings, accompanied by clinical manifestations ranging from headache, altered mental status, seizures, and vision loss, to the loss of consciousness. In 

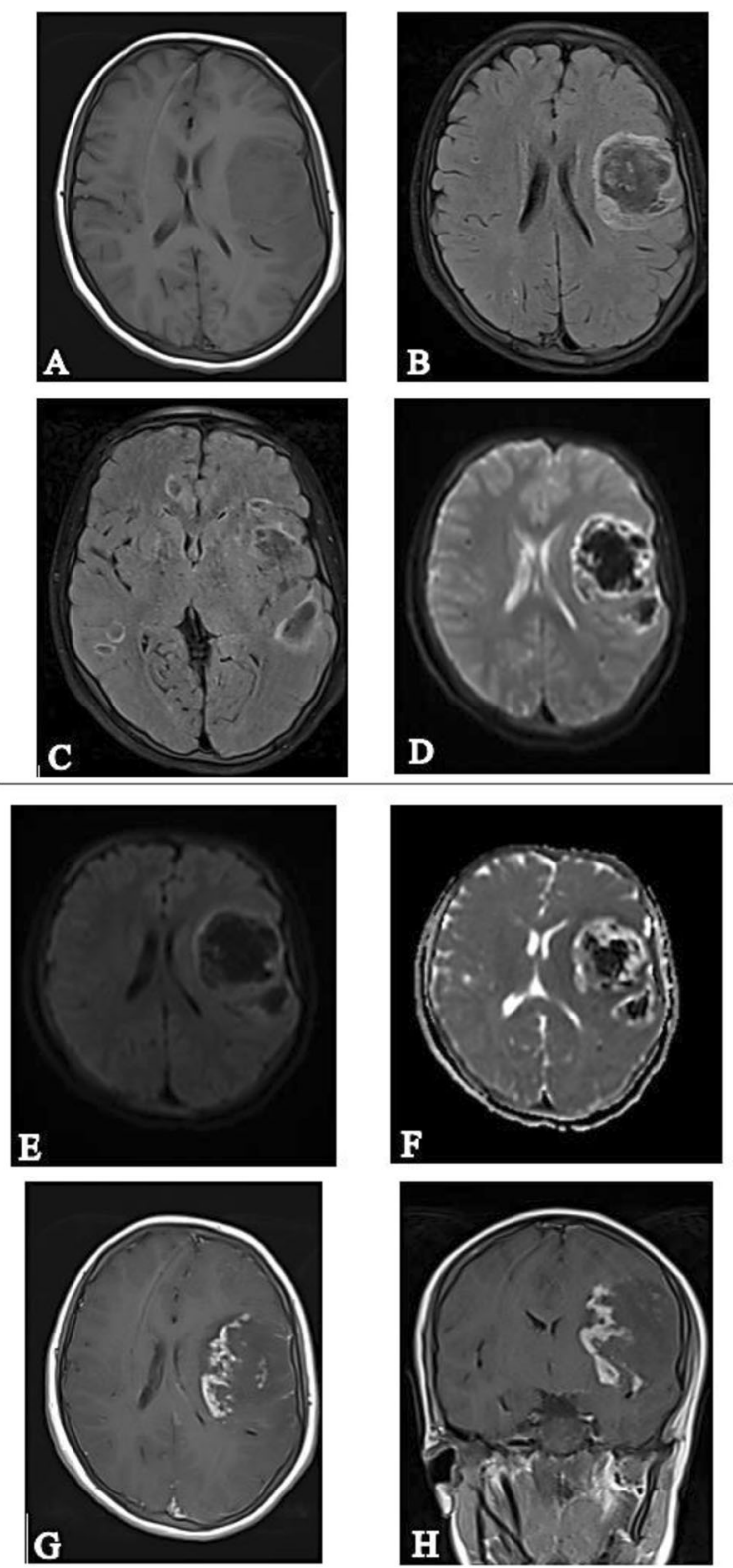

Fig. 8 Multiple intra axial fungomas. Male patient, 8 years old, diagnosed with AML at presentation. Axial MRI of the brain: a Axial T1TW, b, c axial FLAIR images showing left fronto-temporal intra-axial cortical-based SOL elicit intermediate signal in T1WI and Low FLAIR signal with rim of surrounding edema elicits high FLAIR signal. c Image shows other similar smaller lesions seen at both frontal and temporal regions. $\mathbf{d}$ Axial T2*, e axial DWI, and $\mathbf{f}$ axial ADC show blooming of the center of the lesions seen at $\mathrm{T}^{*}$ gradient image (d) with marginal diffusion restriction in DWI (e) and ADC map (f). The low signal in the center of the lesion may be corresponding to ferromagnetic fungal deposits (iron, manganese, magnesium, zinc). g Axial T1 post-contrast MRI. $\mathbf{h}$ Coronal post-contrast MRI of the same patient shows heterogeneous peripheral enhancement of the lesions

the current study, most cases diagnosed with PRES (70\%) were detected early in the induction phase, while $10 \%$ in the consolidation phase, $10 \%$ in the maintenance
Table 4 Correlation between type of leukemia and rate of infiltration

\begin{tabular}{lll}
\hline Type of leukemia & Number of cases with infiltration & Percentage \\
\hline ALL & 7 & $63 \%$ \\
Burkitt's leukemia & 2 & $18 \%$ \\
AML & 1 & $9 \%$ \\
CML & 1 & $9 \%$ \\
\hline
\end{tabular}

phase, and $10 \%$ in the re-induction phase post-relapse. This agreed with Bianca et al. [12] and Raman [13] which have reported that cases with PRES tend to occur early in the induction phase. In the present study, MRI examinations showed typical features of PRES with the most frequently affected areas of the brain being the posterior regions, the occipital and posterior parietal regions $(80 \%)$, followed by the frontal regions $(60 \%)$, and the least commonly affected regions such as the cerebellum, thalami, basal ganglia, and pons which represented $10 \%$ per each. This matched with the study performed by Appachu et al. [14] and in accordance with the findings of the previous studies by Raman [13], Kastrup et al. [15], and McKinney [16].

Although hypertension is a risk factor for PRES, the blood pressure can be normal, particularly in the settings of chemotherapy and immunosuppressive therapy [13]. This is strongly supported by the study results, as most of the studied cases were normotensive apart from only two cases which had low blood pressure.

In the present study, we had two cases with hemorrhage: one had a frontal hematoma, and the other had parenchymal microbleeds. The MRI accurately detected parenchymal hematoma and microbleeds in the two cases. T2* images show high sensitivity in the detection of parenchymal microbleeds. This agreed with the study performed by Charidimou [17] that said the introduction of blood-sensitive MRI sequences, including T2\%-GRE and susceptibility-weighted imaging (SWI), has enabled the accurate detection of cerebral microbleeds (CMBsO) (defined radiologically as small, rounded, homogeneous, hypointense lesions not seen with conventional spin-echo sequences). Also, the study by Beavers et al. [18] stated that GRE images were the most sensitive for the detection of retinal hemorrhages.

Another complication was cerebral infection. It represented $4 \%$ and was detected in two cases of the present study. Both cases had cerebral aspergillosis: one presented with multiple intra-axial masses, and the other had a solitary lesion at the midbrain. Both cases had AML in the early induction phase. The diagnosis of aspergillosis was confirmed by biopsy and detecting galactomannan antigens both in CSF and serum. One of the two patients with multiple masses unfortunately died, and the other patient improved with antifungal 

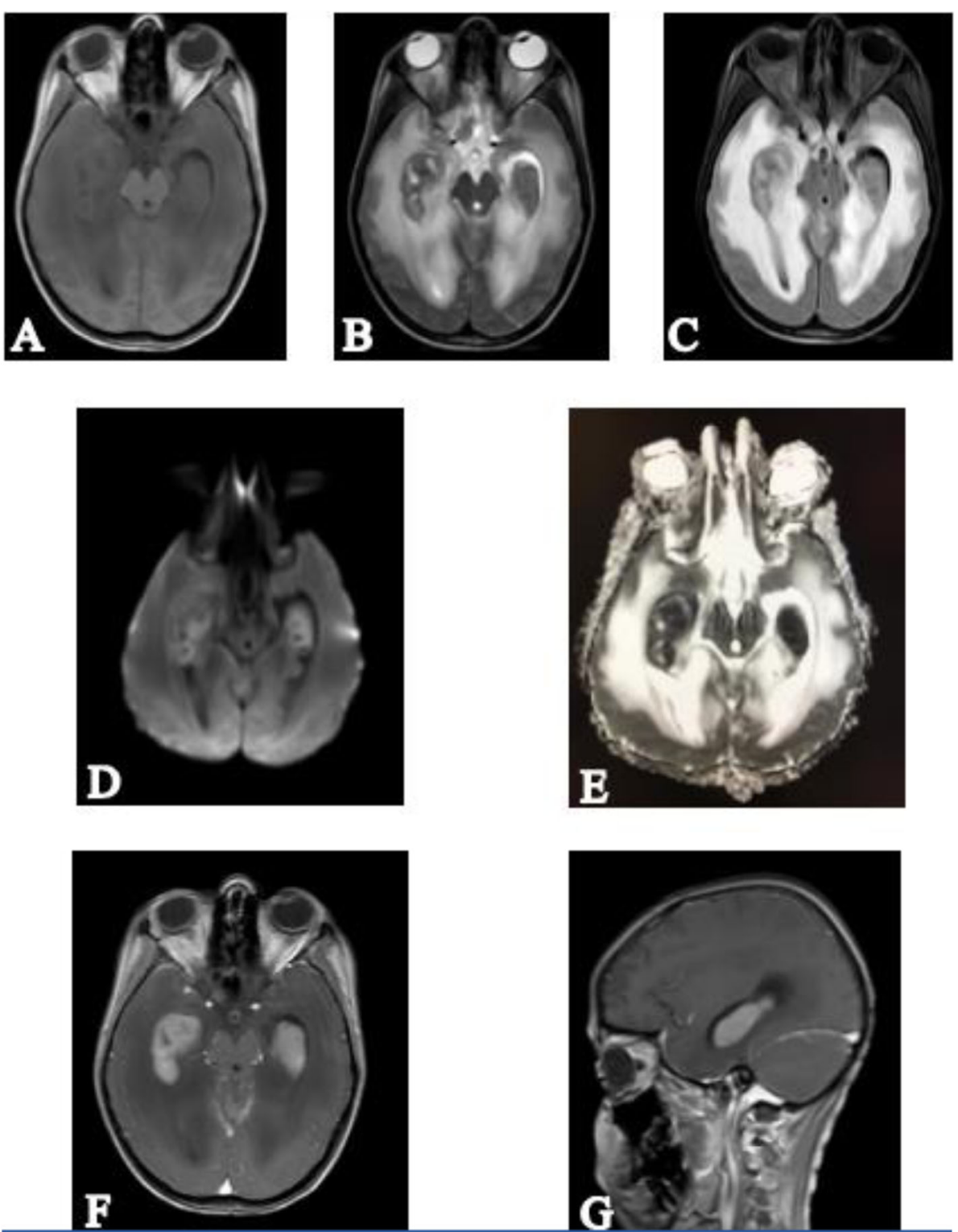

Fig. 9 Leptomeningeal infiltration. Male patient, 15 years old, diagnosed with ALL ended treatment. Axial MRI images of the brain at the level of tempro-occipital region: $\mathbf{a}$ axial non-contrast T1WI, $\mathbf{b}$ axial T2WI, and $\mathbf{c}$ axial FLAIR images show bilateral intra ventricular masses seen filling the temporal horns of both lateral ventricles intermediate signal in T1WI and faint bright signal in T2WI and FLAIR images. Significant periventricular edema is seen eliciting high FLAIR signal. Axial DWI (d) and ADC image (e) show diffusion restriction of intra-ventricular masses. $\mathbf{f}, \mathbf{g}$ Images axial (f) and sagittal (g) post-contrast-enhanced MRI of the brain show avid enhancement of the intra-ventricular masses

Table 5 Site of infiltration in correlation to phase of treatment

\begin{tabular}{llllll}
\hline $\begin{array}{l}\text { Phase of treatment } \\
\text { Site of infiltration }\end{array}$ & Induction & Consolidation & Maintenance & $\begin{array}{l}\text { At } \\
\text { presentation }\end{array}$ \\
\hline Retinal & 1 & - & - & 1 & Relapse \\
Cerebral & 1 & - & - & - & 1 \\
Skull and maxillofacial & - & - & - & 2 & - \\
Meningeal & - & - & - & 1 & - \\
Orbital mass & - & - & - & 1 \\
\hline
\end{tabular}



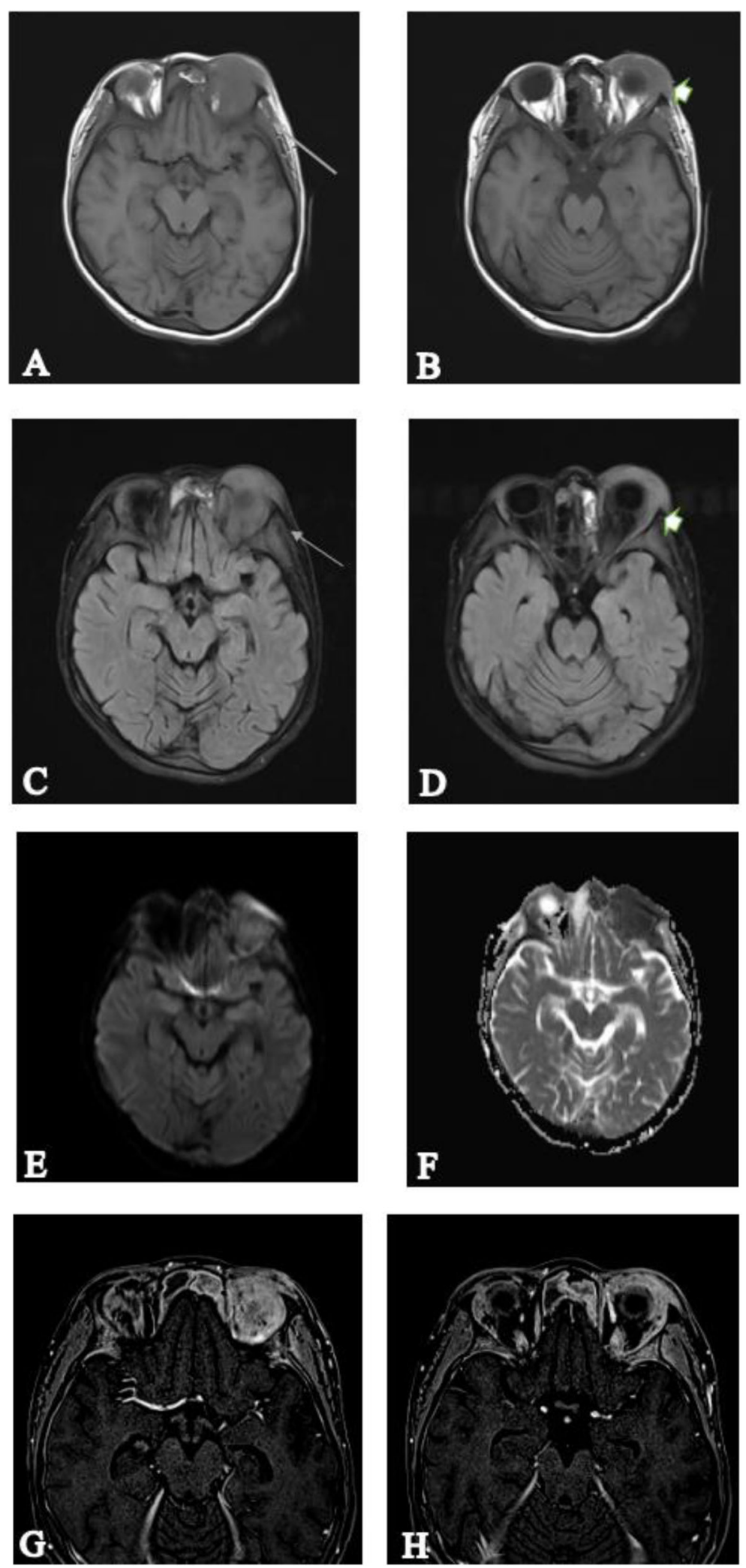

Fig. 10 (See legend on next page.) 
(See figure on previous page.)

Fig. 10 Left orbital leukemic infiltration. Female patient 17 years old diagnosed as Burkitt's leukemia presented by left orbital swelling. Axial MRI of the brain: $\mathbf{a}, \mathbf{b}$ Non-contrast T1WI and $\mathbf{c}, \mathbf{d}$ axial FLAIR images show left intra-orbital mass infiltrating the superior rectus muscle (arrow) and extending to the pre-septal space (thick short arrow). The mass is of intermediate signal in T1WI and bright signal in FLAIR images. e DWI and $\mathbf{f}$ ADC of the same patient show diffusion restriction of the orbital mass. $\mathbf{g}, \mathbf{h}$ Axial post-contrast MRI of the orbit with the patient with fat saturation shows avid enhancement of left orbital mass

treatment. A study performed by Pandian et al. [19] stated that one of the major challenges in AML induction chemotherapy is the risk of death named induction mortality. One of the reasons which place AML patients at risk of acquiring various infections even before initiating induction chemotherapy could be the variable delay in seeking treatment. It is a well-known fact that the incidence and severity of infection depend on the duration of neutropenia. When the neutropenia is prolonged for more than 5 weeks, the chance of infection can be as high as $100 \%$. The signal criteria show characteristic low signal foci with blooming artifacts in the wall and in the core of the lesions at gradient images corresponded to other prior studies by Marzolf et al. [20], Finelli et al. [21], and Luthra [22] which have assumed that this signal could be the result of either ferromagnetic fungal deposits (iron, manganese, magnesium, zinc) or the presence of methemoglobin (in the capsule wall and/or the macrophages) or the presence of free radicals produced by the macrophages.

Previous clinical studies by Marzolf et al. [20] and Gartner et al. [23] had suggested that doctors should exclude the presence of coexisting pulmonary or sinus infections. In the current study, CT examinations of the chest and sinuses were performed in both cases to exclude pulmonary and sinus aspergillosis, and they were positive.

Among the studied patients, 11 cases (22\%) had CNS infiltration with leukemia whether cerebral, retinal, leptomeningeal, or craniofacial bone leukemic infiltration. In the present study, CNS infiltration was common in cases with ALL (63\%), followed by Burkitt's leukemia (18\%), and then CML and AML (each represented 9\%). This correlated with multiple studies by Charles et al.
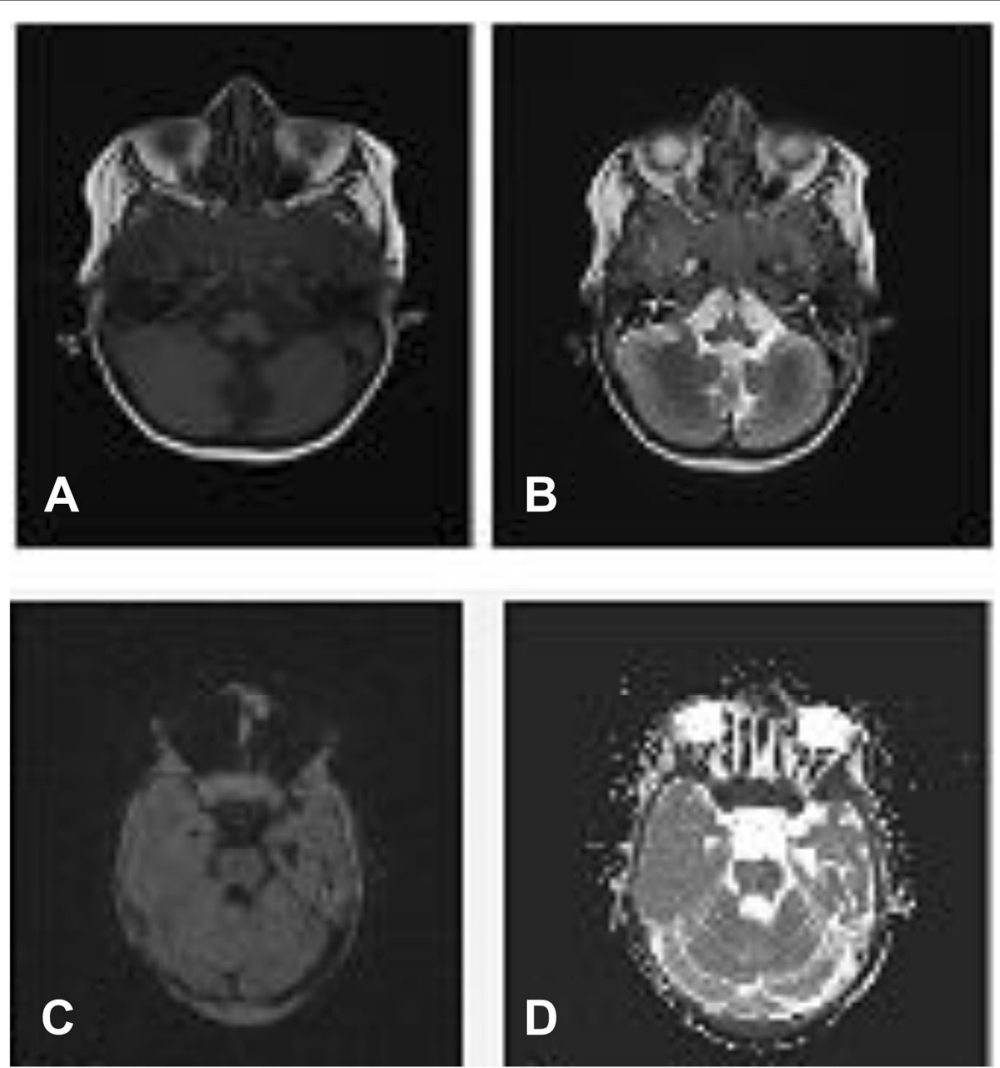

Fig. 11 Skull bone infiltration. Male patient, 7 years old, diagnosed with ALL seen initially at presentation. Axial MRI images of the brain at the level of skull base: $\mathbf{a}$ axial non-contrast T1WI, $\mathbf{b}$ axial T2WI, $\mathbf{c}$ axial DWI, and $\mathbf{d}$ axial ADC images show diffusion bone marrow infiltration with cortical expansion and diffusion restriction in DWI and ADC map 

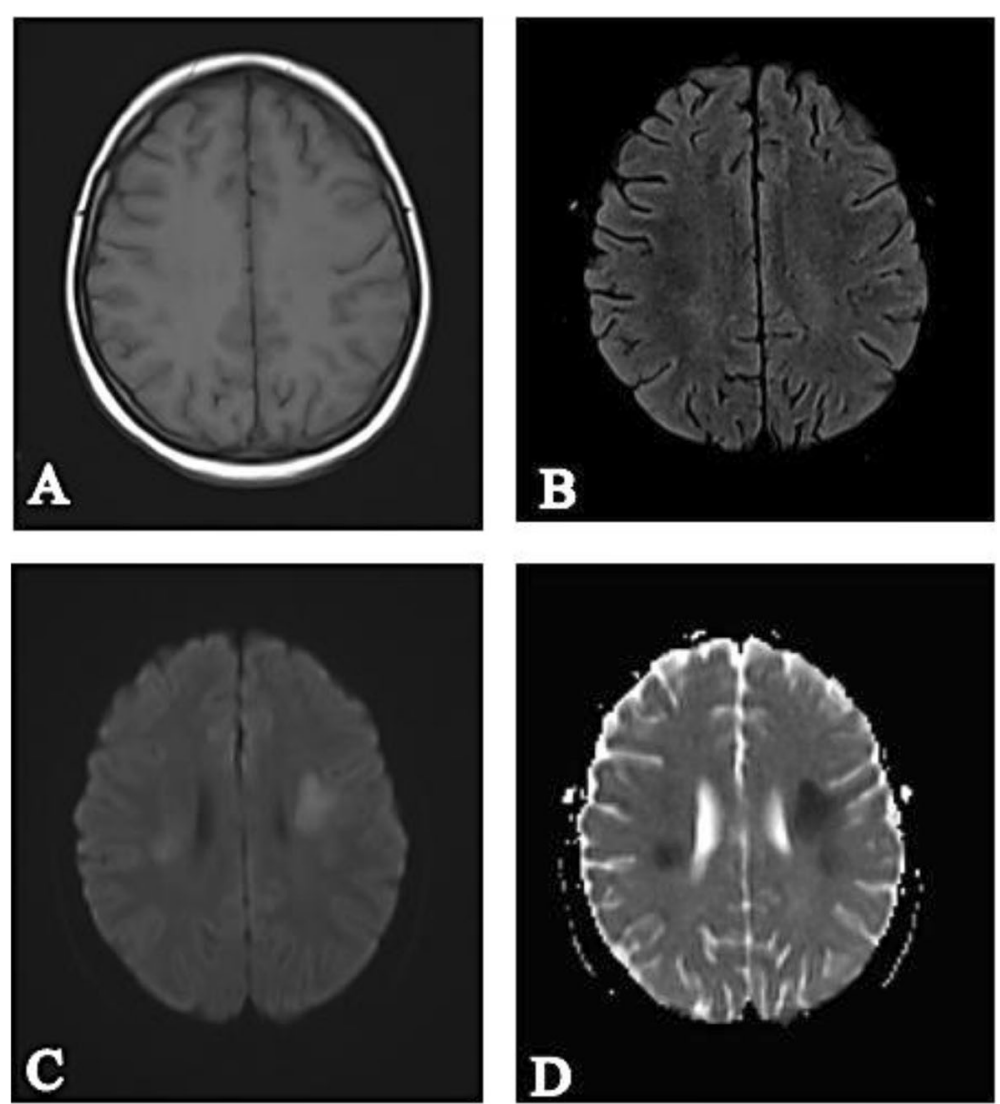

Fig. 12 MTX toxicity. Female patient, 15 years old, diagnosed with ALL at consolidation phase on methotrexate therapy. Axial MRI images of the brain at the level of fronto-parietal regions. The $\mathbf{a}$ TIWI, $\mathbf{b}$ FLAIR, $\mathbf{c}$ DWI, and $\mathbf{d}$ ADC map shows bilateral periventricular areas of diffusion restriction with no gross signal abnormalities seen at conventional T1WI or FLAIR images

[24] and Del Principe et al. [25] which stated that CNS involvement in patients with AML is considerably less common than CNS involvement by acute lymphoblastic leukemia (ALL) in both adults and children.

In the present study, meningeal infiltration was detected in three cases. The first case showed enhanced dural thickening and subdural collection which were reported as inflammatory versus infiltration. CSF cytology revealed blast cells in CSF. The second showed multiple meningeal-based enhanced small masses at the brain and spine which were reported as infiltration. The last one showed diffuse ependymal enhancement and intraventricular masses. These cases were proved by CSF analysis and showed regression in the follow-up studies post-treatment with chemotherapy.

Cerebral parenchymal involvement was detected in two cases and appeared as multiple enhancing foci and illdefined areas seen at the thalami, brain stem, and cerebellum which were reported as inflammatory versus infiltration. Further CSF analysis proved leukemic infiltration.

Among the cases of leukemic infiltration, there was one case with Burkitt's leukemia that had a left orbital mass, proved by biopsy as leukemic infiltration. The MRI findings showed imaging characteristics that were compatible with those described by other studies [26-29].

In the present study, MRI had high sensitivity in the detection of retinal involvement in all three cases presented by focal retinal enhancement and thickening with enhanced nodules and diffusion restriction in DWI. This was proved by ophthalmic examination which showed subretinal fluid collection suggestive of infiltration. The follow-up studies showed marked improvement postinduction of chemotherapy. These findings corresponded to a study performed by Green et al. [30] and Pflugrath [31].

In the current study, MRI had detected bone marrow infiltration of the skull and maxillofacial bones in two cases by alteration of the marrow signal, cortical expansion, erosions, and associated soft tissue masses that showed post-contrast enhancement and diffusion restriction in DWI. This agreed with a study performed by Eisa [32] and Cao et al. [33] which said that MRI with DWI is a useful and non-invasive tool for detecting skull bone infiltration in ALL children before treatment and also the normalization of marrow signal at complete remission after therapy. 
Toxicity related to high-dose methotrexate was detected in five patients presented by bilateral basal ganglia, periventricular regions, and centrum semi-oval areas with diffusion restriction and low ADC value. Subtle or no abnormal FLAIR signal was noted. These findings were similar to those described by Cruz-Carreras et al. [34] and Tando [35], as well as a study by Michael et al. [36] which concluded that DWI has the potential role for early detection of methotrexate white matter injury.

Periventricular leukoencephalopathy was detected in one case at the maintenance phase, presented by a periventricular deep white matter sheet of high FLAIR signal which was constant in follow-up studies and one case with atrophic brain changes post-end of therapy manifested by decreased cerebral volume and widened extraaxial CSF spaces. Both cases had no history of cranial irradiation. In a study performed by Porto et al. [9] said that atrophy of the brain was believed to be a late finding after irradiation related to a diffuse white matter injury, but other studies have suggested that the atrophic changes may be related to chemotherapy, with cranial irradiation playing a lesser role.

Mineralizing angiopathy was present in one case, which appeared as multiple calcific foci at the gray-white mater interface; this case had history of cranial radiation before the development of these calcifications. Calcifications were seen easily in non-contrast $\mathrm{CT}$, while in MRI showed a faint bright signal at T1WI and a low signal at T2WI. This agreed with a study performed by Rossi [37] which stated that the best diagnostic radiologic tool for mineralizing microangiopathy is $\mathrm{CT}$, which shows calcium deposition mainly localized in the basal ganglia and subcortical white matter.

\section{Conclusion}

The neurological complications of leukemia have common presenting symptoms but varying imaging abnormalities. To reach the correct diagnosis, the presenting signs, symptoms, and laboratory data must be considered along with the radiologic findings. A diagnostic algorithm using conventional, post-contrast MRI, MR venography, along with diffusion-weighted MRI was of great value in early detection and differentiation of different CNS lesions detected in pediatric patients with leukemia and post-treatment CNS complications.

\section{Abbreviations}

MRI: Magnetic resonance imaging; CNS: Central nervous system; DWI: Diffusion-weighted imaging; ADC: Apparent diffusion coefficient; PRES: Posterior reversible encephalopathy syndrome; MTX: Methotrexate

\section{Acknowledgments}

I would like to record my appreciation to Dr. Mohamed Samy Said El-Azab MD radio diagnosis and intervention radiology in National Cancer Institute, Cairo University, Cairo, Egypt, for his help and encouragement.

\section{Authors' contributions}

EM wrote the manuscript and collected the patient data and is responsible for the correspondence to the journal. AF is the author of the research idea. MR participated in the design and review of the study. AY participated in the design and review of the study. HK participated in the design and review of the study. The authors read and approved the final manuscript.

Funding

Not applicable.

\section{Availability of data and materials}

The datasets used and analyzed during the current study are available from the corresponding author on reasonable request.

\section{Ethics approval and consent to participate}

The study was approved by the Department of Radiology, the Banha Faculty of Medicine Research Ethics Committee, University of Banha, Egypt (ethics committee reference number is not available. Our committee is going to settle a number in the future, but now it is not available). A written consent was obtained from each child's guardian involved in this research before performing the study.

\section{Consent for publication}

Images included in the study are entirely unidentifiable, and there are no details on individuals reported within the manuscript.

\section{Competing interests}

The authors declare that they have no competing interests.

\section{Author details}

${ }^{1}$ Tanta Cancer Center, El Nadi Street, Tanta, Egypt. ${ }^{2}$ Radiology Department, Banha University, Banha, Egypt. ${ }^{3}$ Radiology Department, Helwan University, Helwan, Egypt. ${ }^{4}$ Radiology Department, National Cancer Institute, Cairo University, Cairo, Egypt.

Received: 11 August 2020 Accepted: 19 October 2020

Published online: 04 November 2020

\section{References}

1. Pahloosye A, Hashemi A, Mirmohammadi SJ et al (2011) Presenting clinical and laboratory data of childhood acute lymphoblastic leukemia. Iran J Ped Hematol Oncol 1(3):71-77.

2. Verma A, Manisha R, Verma M et al (2018) Spectrum of clinical presentations of acute leukemia tertiary care hospital of Jharkhand. Int J Curr Innov Res 4(A):1159-1161

3. Taner A, Anil O, Tugana A et al (2017) Magnetic resonance imaging of neurologic complications through the treatment of childhood leukaemia and lymphoma. Can Assoc Radiol J 68(2):98-105

4. Kembhavi SA, Somvanshi S, Banavali S et al (2012) Pictorial essay: acute neurological complications in children with acute lymphoblastic leukemia. Indian J Radiol Imaging 22(2):98-105

5. Ulu EM, Töre HG, Bayrak A et al (2009) MRI of central nervous system abnormalities in childhood leukemia. Diagn Interv Radiol 15(2):86-92

6. Jing L, Yaming W, Xuefei S et al (2017) Lesions of the central nervous system in leukemia: Pathologic and magnetic resonance imaging features at presentation in 14 patients. Oncol Lett 14:8162-8170

7. Terwilliger T, Abdul-Hay M (2017) Acute lymphoblastic leukemia: a comprehensive review and 2017 update. Blood Cancer J 7(6):e577

8. Malhotra P, Jain S, Kapoor G (2018) Symptomatic cerebral sinovenous thrombosis associated with $\mathrm{L}$-asparaginase in children with acute lymphoblastic leukemia: a single institution experience over 17 years. J Pediatr Hematol Oncol 40(7):e450-e453

9. Porto L, Kieslich M, Schwabe D et al (2004) Central nervous system imaging in childhood leukaemia. Eur J Cancer 40(14):2082-2090

10. Vázquez E, Lucaya J, Castellote A et al (2002) Neuroimaging in pediatric leukemia and lymphoma: differential diagnosis. Radiographics 22(6): 1411-1428

11. Vazquez E, Delgado I, Sanchez A et al (2011) Enriquez G. Side effects of oncologic therapies in the pediatric central nervous system: update on neuroimaging findings. Radiographics 31(4):1123-1139 
12. Bianca P, Annemarie M, Gijs TJ et al (2010) Posterior reversible encephalopathy syndrome in paediatric leukaemia. Eur J Paediatr Neurol: 539-545

13. Raman R, Devaramane $R$, Jagadish GM et al (2017) Various imaging manifestations of posterior reversible encephalopathy syndrome (PRES) on magnetic resonance imaging (MRI). Pol J Radiol 82:64

14. Appachu MS, Purohit S, Lakshmaiah KC et al (2014) Posterior reversible encephalopathy syndrome in pediatric acute leukemia: case series and literature review. Indian J Med Paediatr Oncol 35(1):79

15. Kastrup O, Schlamann M, Moenninghoff C et al (2015) Posterior reversible encephalopathy syndrome: the spectrum of MR imaging patterns. Clin Neuroradiol 25(2):161-171

16. McKinney AM, Short J, Truwit CL et al (2007) Posterior reversible encephalopathy syndrome: incidence of atypical regions of involvement and imaging findings. Am J Roentgenol 189(4):904-912

17. Charidimou A, Shakeshaft C, Werring DJ (2012) Cerebral microbleeds on magnetic resonance imaging and anticoagulant-associated intracerebral hemorrhage risk. Front Neurol 3:133

18. Beavers AJ, Stagner AM, Allbery SM et al (2015) MR detection of retinal hemorrhages: correlation with graded ophthalmologic exam. Pediatr Radiol 45(9):1363-1371

19. Pandian J, Raghavan V, Manuprasad A et al (2020) Infection at diagnosis-a unique challenge in acute myeloid leukemia treatment in developing world. Support Care Cancer:1-6

20. Marzolf G, Sabou M, Lannes B et al (2016) Magnetic resonance imaging of cerebral aspergillosis: imaging and pathological correlations. PLoS One 11(4):e0152475

21. Finelli PF, Foxman EB (2014) The etiology of ring lesions on diffusionweighted imaging. Neuroradiol J 27(3):280-287

22. Luthra G, Parihar A, Nath $\mathrm{K}$ et al (2007) Comparative evaluation of fungal, tubercular, and pyogenic brain abscesses with conventional and diffusion MR imaging and proton MR spectroscopy. AJNR Am J Neuroradiol 28:1332-1338

23. Gartner F, Forstenpointner J, Ertl-Wagner B et al (2018) CT and MRI findings in cerebral aspergilloma. Röfo 190(10):967-970

24. Charles AS, Richard AL and Alan GR (2020): Involvement of the central nervous system with acute myeloid leukemia. UpToDate.

25. Del Principe MI, Buccisano F, Soddu S et al (2018) Involvement of central nervous system in adult patients with acute myeloid leukemia: Incidence and impact on outcome. Semin Hematol 55:209

26. Keraliya AR, Krajewski KM, Giardino AA et al (2015) Imaging of nervous system involvement in hematologic malignancies: what radiologists need to know? Am J Roentgenol 205(3):604-617

27. Shen $H$, Zhao $Y$, Shi $Y$ et al (2020) The diagnostic and prognostic value of MRI in central nervous system involvement of acute myeloid leukemia: a retrospective cohort of 84 patients. Hematology 25(1):258-263

28. Bidar M, Wilson MW, Laquis SJ et al (2007) Clinical and imaging characteristics of orbital leukemic tumors. Ophthalmic Plast Reconstr Surg 23(2):87-93

29. Grasso D, Borreggine C, Ladogana S et al (2016) Sporadic Burkitt's lymphoma/acute B-cell leukaemia presenting with progressive proptosis and orbital mass in a child. Neuroradiol J 29(3):231-235

30. Green W, Rao PK, Harocopos GJ (2017) Extramedullary relapse of acute myelogenous leukemia presenting as a large serous retinal detachment. Ocul Oncol Pathol 3(2):95-100

31. Pflugrath $A E$, Brar VS (2020) Bilateral optic nerve and retinal infiltration as an initial site of relapse in a child with T-cell acute lymphoblastic leukemia. Am J Ophthalmol Case Rep 18:100695

32. Eisa HM, Gamal El Deen MM, Abdullah YA et al (2017) Role of MRI in the diagnosis of different bone marrow lesions in pediatric patients with hematological malignancies. Egypt J Hosp Med 69(2):1889-1894

33. Cao W, Liang C, Gen Y et al (2016) Role of diffusion-weighted imaging for detecting bone marrow infiltration in skull in children with acute lymphoblastic leukemia. Diagn Interv Radiol 22(6):580-586

34. Cruz-Carreras MT, Chaftari P, Shamsnia A et al (2017) Methotrexate-induced leukoencephalopathy presenting as stroke in the emergency department. Clin Case Rep 5(10):1644-1648

35. Tandon N, Khanderia M, Devadas SK (2019) Panda eye sign on diffusion weighted-MRI for the diagnosis of methotrexate-induced subacute leukoencephalopathy. Cancer Res Stat Treat 2(2):263
36. Michael J, Zarir P, Erin M et al (2005) Diffusion-weighted MR imaging of early methotrexate-related neurotoxicity in children. Am J Neuroradiol 26(7):1686-1689

37. Rossi Espagnet MC, Pasquini L, Napolitano A et al (2017) Magnetic resonance imaging patterns of treatment-related toxicity in the pediatric brain: an update and review of the literature. Pediatr Radiol 47:633-648

\section{Publisher's Note}

Springer Nature remains neutral with regard to jurisdictional claims in published maps and institutional affiliations.

\section{Submit your manuscript to a SpringerOpen ${ }^{\circ}$ journal and benefit from:}

- Convenient online submission

- Rigorous peer review

- Open access: articles freely available online

High visibility within the field

- Retaining the copyright to your article

Submit your next manuscript at $\boldsymbol{\nabla}$ springeropen.com 